\title{
Software Defined Radio Baseband Processing for ESA ESEO Mission
}

\author{
P. Bartram, C. P. Bridges \\ Surrey Space Centre \\ University of Surrey \\ Guildford, Surrey, United Kingdom \\ +44 (0)1483689137 \\ c.p.bridges@surrey.ac.uk
}

\begin{abstract}
The European Student Earth Orbiter (ESEO) is a micro-satellite mission to Low Earth Orbit and is being developed, integrated, and tested by European university students as an ESA Education Office project. AMSAT-UK and Surrey Space Centre are contributing to the mission with a transceiver and transponder similar to that of FUNcube-1 with the addition of utilising a Atmel AT32 processor for packet software-redundancy, baseband processing, forward error correction, and packet forming; acting as a step towards software defined radio using low MIPS automotive microprocessors. As on the FUNcube-1 satellite, the telemetry formats and encoding schemes presented utilize a large ground network of receivers on the VHF downlink and conforms to 1200 bps and a new 4800 bps redundant downlink for the rest of the spacecraft. The uplink is on L-band using bespoke partial-CCSDS frames.
\end{abstract}

This paper details the flight software on the engineering and flight models to ESA, and the technical configuration and associated tests of demonstrating the processor load is under for varying operating and sampling modes. In particular, a key contribution will be the details of utilising the Google Test Suite for verification of the SDR functions and FreeRTOS tools to optimize processor load margins to $30 \%$ when operating parallelized ADC and DAC, and CAN-open telemetry chains and what memory considerations are needed to ensure stable long-term operations.

\section{TABLE OF CONTENTS}

1. ESEO AMSAT-UK PAYLOAD.............................. 1 2. SOFTWARE CHALLENGES .................................... 2 3. VARIABLE BPSK DOWNLINK ................................. 3 4. AFSK UPLINK........................................................... 5 5. THREAD RELIABILITY \& PERFORMANCE.............. 6 6. ProjeCt EXPERIENCES \& ChallengeS.............. 7

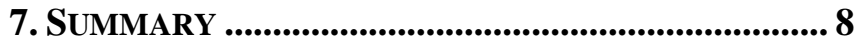
ACKNOWLEDGEMENTS ............................................ 8 REFERENCES........................................................... 8 BIOGRAPHY ........................................................... 9

\section{ESEO AMSAT-UK PAYLOAD}

The European Student Earth Orbiter (ESEO) is a microsatellite mission to low Earth orbit (LEO) being developed, integrated, and tested by European university students as an

\author{
D. Bowman, G. Shirville \\ AMSAT-UK \\ Badgers, Letton Close, \\ Blandford, Dorset \\ DT11 7SS \\ G0MRF@aol.com / G3VZV@amsat.org
}

ESA Education Office project together with an industrial prime contractor called Sitael (formly ALMASpace) in Italy. ESEO aims to provide student payload teams with unparalleled hands-on experience to help prepare a wellqualified space-engineering workforce for Europe's future. The teams are expected to provide spacecraft subsystems, payloads and ground support systems as part of their academic studies; and AMSAT-UK have teamed with Surrey Space Centre at the University of Surrey to deliver an amateur communications payloads which also acts as a redundant downlink.

The development of an amateur communications payload began in 2008 and, at the time, a $5.6 \mathrm{GHz}$ transmitter and UHF receiver was proposed together with a laser beacon. However, the mission was re-evaluated by ESA at Phase B and the payload was revised to minimize spacecraft mass. As such, a VHF transmitter and L-band receiver was proposed which also avoided the primary UHF TMTC transceiver frequencies [1].

To fit the university calendar, the development was completed over a number of years. To meet interface baseband requirements of a transmitter and receiver, dual CANopen and I2C telemetry buses, the Atmel AT32UC3C processor was chosen. The main hardware and software control interfaces were built up for I2C, CANopen, and also a 1200 baud AFSK receiver using open source demodulator C code [2]. As a proof of concept, the initial team of two Brazilian 'Science Without Border' students collected APRS signals from the baseband audio output of an ICOM910H radio and transmitted them over CANopen demonstrating key parts of the chain. These students worked on input and output filters for both hardware and software to receive 1200 baud AFSK and also transmit 1200 baud BPSK. These student engineers improved on the software too by understanding signal quantization, measurement, and phase error to understand where soft and hardware decisions are made in decoders - and by taking a windowed data approach to ensure correct decisions. These plots recorded sampled data and output bits throughout the decoding process for viewing and transferring to computers for analysis; see Figure 1. After this initial concept development, there were two further U.K. students that worked on the Engineering Model (EM) development PCB designs. 


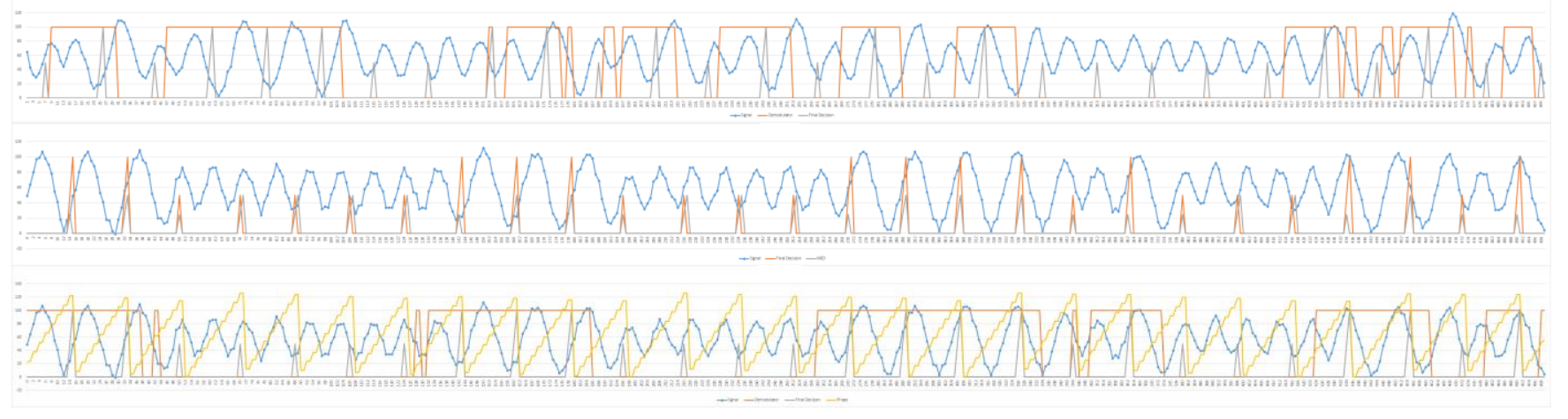

Figure 1: a) Correlating decoder decisions with output samples, b) marking decision points, and c) windowed decision making with phase compensation

The EM is broken into three boards: a computing and electrical power board, an L-band receiver, and a VHF transmitter; see Figure 2.

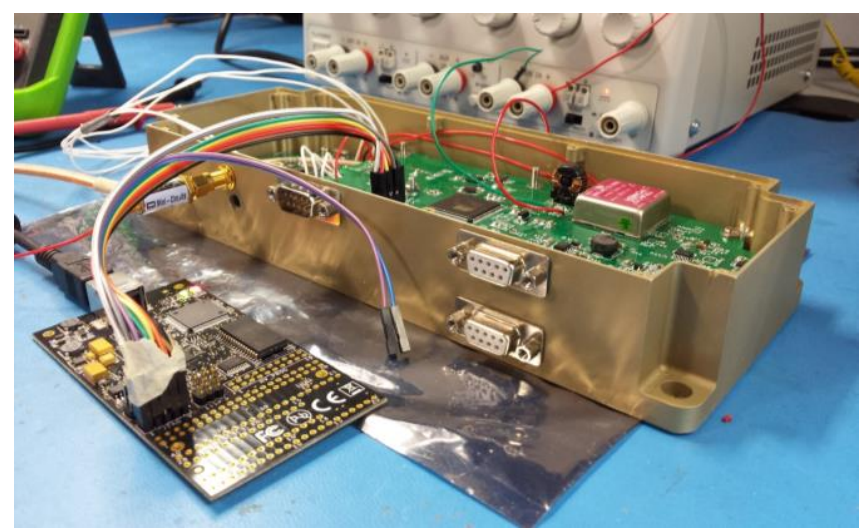

Figure 2: AMSAT Payload during firmware programming prior to testing

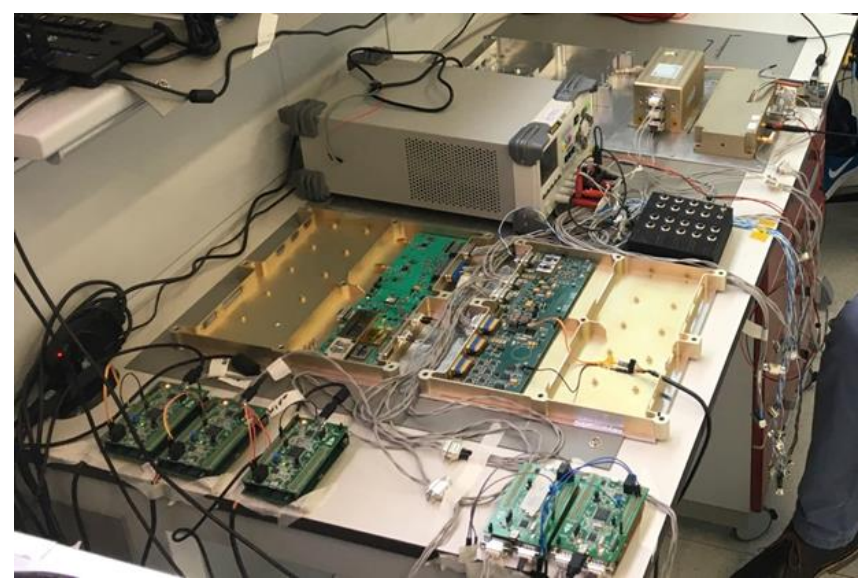

Figure 3: EM during integration \& test at the Sitael Lab Facilities in October 2016 at Forli, Italy (see top-right)

The unit locates the two RF boards underneath the computing and electrical power board which is visible in Figure 2. The external interfaces are dual CAN interfaces, one power connector, two SMA antenna connectors, and one JTAG connector for debugging and programming. In addition to these students, a U.K. MSc student who realized the flight software was involved is the primary focus of this paper. In the future, a German student will aid in the FM unit build and environmental test, qualification, and acceptance.

\section{Software Challenges}

A key finding of the literature reviewed for this project was that in order to meet ESA timing and resource management constraints [3], it would be necessary to utilize a real time operating system (RTOS). Due to its small memory footprint and processor cycle overhead, combined with its free license, FreeRTOS was selected for use. This allowed for functional areas of the software to be split up into threads, thereby isolating discrete software functionality and allowing for best practice development techniques to be used. Therefore CANopen, uplink, downlink, telemetry collection, payload data transfer and satellite operations were split into individual threads.

A problem encountered due to the multi-organizational nature of this project was that of developing software to a strictly defined interface between organizations and proving the functionality before the two systems were coupled. An example of this was the application layer protocols operating on top of the CANopen communications protocol, allowing for communications between the AMSAT payload and satellite itself. An emulator with limited functionality was supplied by ALMAspace to ensure correct hardware configuration however this would not prove higher level software operations, as a trial a software development practice entitled Test Driven Development (TDD) was used throughout the project, the end result of this was that when the payload was integrated with the satellite it worked immediately - the value of TDD has been seen many times over during this development and should be strongly considered by anyone wishing to collaborate on a project successfully.

A key consideration for the development of this payload was the embedded processor memory and processing constraints. The microcontroller was specifically selected to 
have the highest amount of RAM and flash available for its class, with $64 \mathrm{kB}$ and $512 \mathrm{kB}$ respectively. The memory map can be seen in Figure 4 with separate regions being allocated to program data, filter lookup tables, default values and also to payload data. The first two of these regions are fixed after programming whereas the final two regions are dynamic as the mission progresses, therefore the lower two regions were locked during programming to ensure that a single event upset (SEU) could not cause a write meant for the dynamic region to be redirected to overwrite the fixed region - potentially causing catastrophic mission failure.

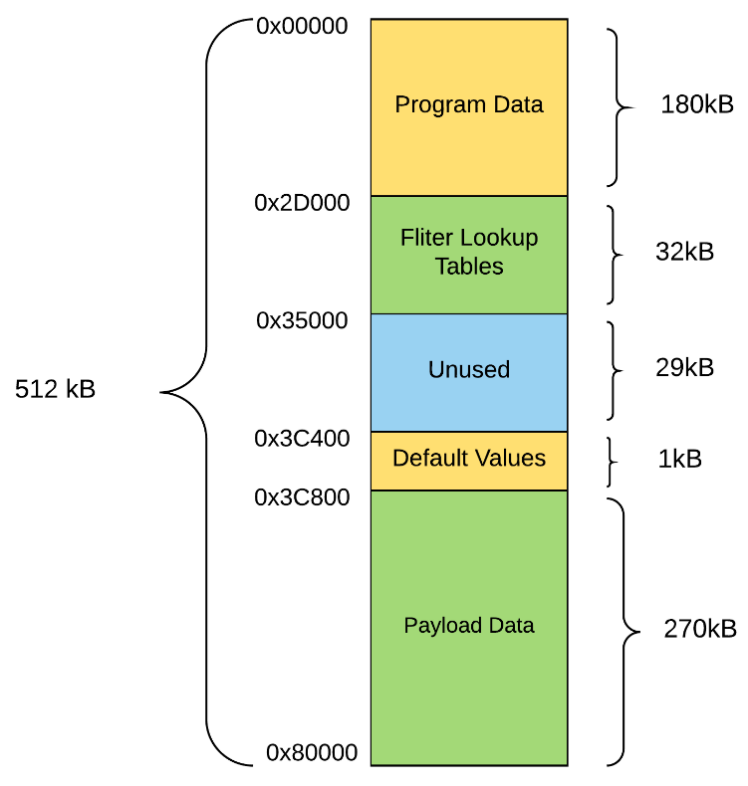

Figure 4: AT32 Memory Map

SEUs are a major cause of concern for non-radiation harderned devices and without writing a large amount of protective code it is difficult to protect against their effects. In addition, it is possible that the protective code can also inadvertently introduce bugs, therefore a non-code-invasive method of protecting against a percentage of SEUs was desirable.

A novel method for protection was therefore trialed by making modifications to the FreeRTOS kernel itself, and combined the traditional protective method of triple modular redundancy (TMR) of data with the FreeRTOS context switching system. Thus, whenever a context switch is made by FreeRTOS the kernel modifications ensure that no SEUs have caused bit flips in the context stack about to be switched in - this offers protection to all variables placed onto the stack, and offers a protection percentage inversely proportional to the percentage of time that a thread is running. The overhead associated with this protection process is clearly application specific and depends on the amount of data placed onto the stack and also on whether threads are preempted or not. Application specific speed gains can be achieved through only checking variables on the stack that are currently active. In the case of this payload this technique offered protection against $27 \%$ - $99 \%$ of SEUs depending on the particular thread at a processor overhead of $\sim 5 \%$. SEUs detected and corrected via the use of this technique are transmitted in the satellite telemetry package and therefore an evaluation of the efficacy will later be performed with on-orbit data.

\section{VARIABLE BPSK DOWNLINK}

The primary mission of this payload is the collection and transmission of telemetry data to the ground. However, in the event of failure of the primary communications system, this payload must also act as a redundant downlink option for transfer of scientific data from other scientific payloads. Therefore it was necessary for the payload to be able to transfer telemetry and scientific data reliably, without error and at a rate that allows for scientific data to be transferred in a 'reasonable' time period, reasonable was defined as being within a typical LEO groundstation pass $\sim 7$ minutes. Such that the payload could make use of the AMSAT groundstation network, it was necessary to conform to the AO-40 [4] forward error correction (FEC) standard, this was done simply through the use of a $\mathrm{C}$ library - the only functional restriction this imposed was that a packet of 256 bytes had to be used which would then grow in size to 650 bytes including forward error correction bits.

Standard downlink packets are split into two parts, a real time telemetry (RTT) section and a whole orbit data (WOD) section, but ultimately the sum of these two parts is 256 bytes with the RTT being sampled via the I2C sensors every 5 seconds. Therefore a data rate of $1.2 \mathrm{kbps}$ allows for the RTT to be transmitted in realtime - taking 4.3 seconds to transmit once the FEC data is added. In contrast scientific data transfer was not capable of being transferred at this data rate, with a maximum payload data size of $64 \mathrm{kB}$ requiring $\sim 18$ minutes after FEC data is added. This is longer than a typical LEO groundstation pass and was therefore unacceptable. Thus an increased data rate of 4.8 kbps was developed to bring the transfer time to within the desired window. Both data rates were implemented using a BPSK modulation scheme to increase the reliability over a lossy link and be spectrally efficient.

As the modulation scheme and two data transmission rates were known, it was necessary to determine the rate at which the DAC would output samples to the modulator. The ratio of the sampling rate to the BPSK baud rate is the number of DAC output samples representing a single symbol, and is commonly referred to as the DAC oversampling rate. Theoretically, it is possible to reconstruct an identical DAC output waveform perfectly so long as the oversampling rate is equal to the Nyquist frequency, i.e twice the highest frequency component [5]. However, sampling at this rate means that the closest signal harmonics image will be present at twice the signal frequency [6] and adds severe requirements on the roll-off of the hardware anti-aliasing filter in order to stop transmission of these higher frequency components. Therefore, it was decided that oversampling would be applied to reduce the requirements 
on this filter. Traditional systems such as CD players use an oversampling rate of 2, 4 or 8 times the Nyquist frequency [6], therefore this served as a starting point from which to evaluate the performance at these rates. For software design simplicity, a single DAC sampling rate was used for both transmission modes. A final sampling rate of $19.2 \mathrm{ksps}$ was used, meaning the $1.2 \mathrm{kbps}$ and $4.8 \mathrm{kbps}$ signals were oversampled at eight times and twice the Nyquist frequency respectively. Another consideration was that of the trade-off between sampling rate impact on waveform quality and the processor cycles available to perform other operations between samples. A sampling rate of $19.2 \mathrm{ksps}$ allowed for 3125 processor operations to be performed between each sample, allowing for data to be encoded and delivered to the DAC at the correct time.

As an initial experiment, an unfiltered output signal was fed into the BPSK modulator and the broadcast RF signal observed. The frequency domain result of this can be seen in Figure 5. It can clearly be seen that there are signal harmonics present, with the highest harmonic amplitude being only $-15 \mathrm{~dB}$ below the intended signal even with a hardware low pass filter present.

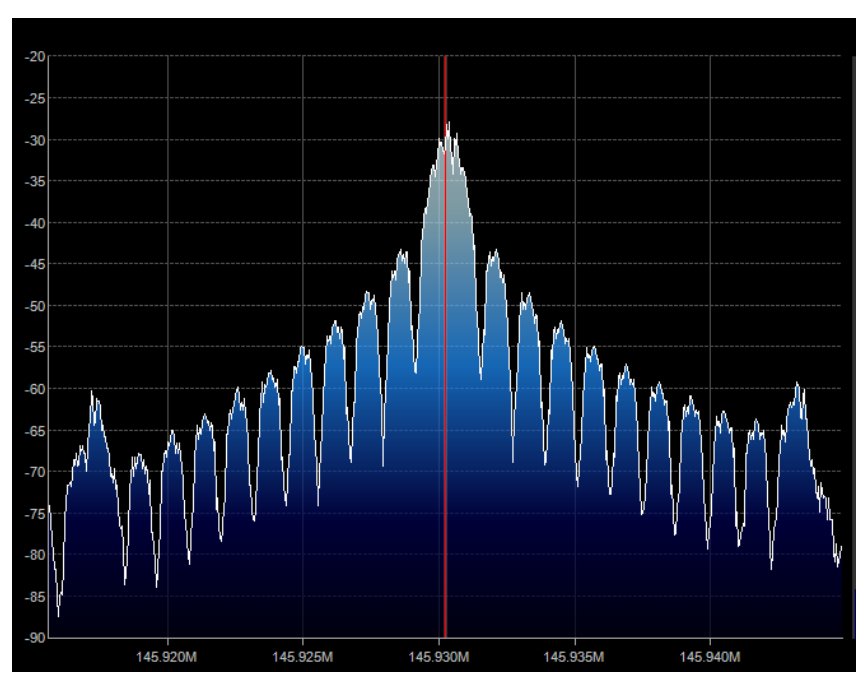

Figure 5: Unfiltered BPSK modulator output

This was unacceptable and further digital signal processing was applied to filter the output waveform. This was done through the use of the Raised Root Cosine (RRC) Filter and is specified through Equation 1 [7].

$$
\begin{aligned}
& H_{R C F} \\
& =\left\{\begin{array}{lr}
T, & |f| \leq \frac{1-\beta}{2 T} \\
\frac{T}{2}\left[1+\cos \left[\frac{\pi T}{\beta}[|f|]-\frac{1-\beta}{2 T}\right]\right], & \frac{1-\beta}{2 T}<|f| \leq \frac{(1+\beta)}{2 T} \\
0, & \frac{1+\beta}{2 T}<|f|
\end{array}\right.
\end{aligned}
$$

Matlab was used with (1) to generate impulse and frequency response graphs and prove the filter was fit for purpose, these can be seen for the $4.8 \mathrm{kHz}$ data rate in Figure 6 and Figure 7 respectively.

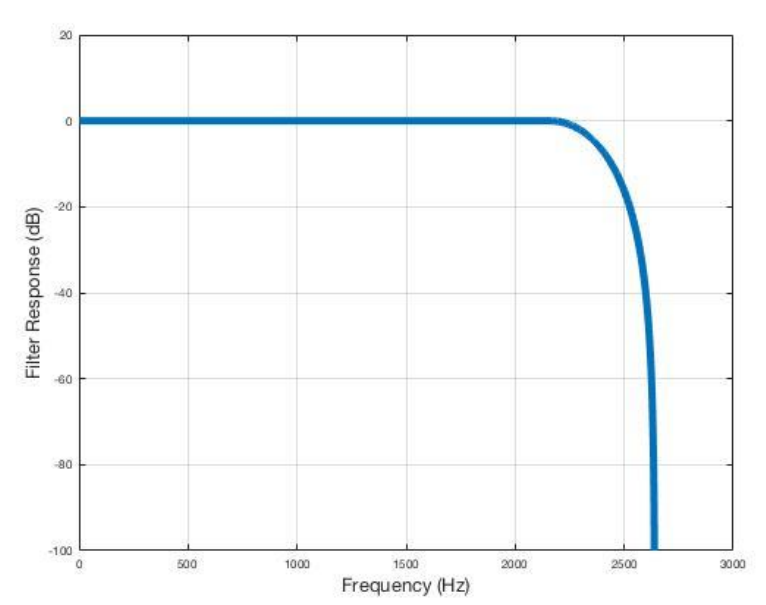

Figure 6: 4k8 Filter frequency response

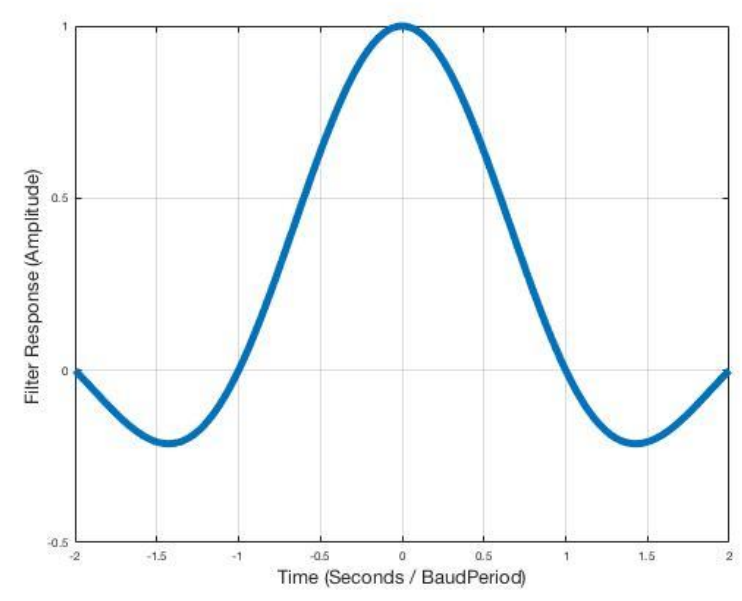

Figure 7: 4k8 Filter time response

In order to apply the RRC filter to a given signal, convolution was considered as an option. It was found that for low numbers of filter coefficients $(<\sim 130)$ this is a faster process than performing Fast Fourier Transforms (FFTs). Convolution as a process requires multiplication and accumulation of the output signal with the filter impulse response coefficients, therefore it is possible to calculate the processing requirements. Assuming 130 filter coefficients and a DAC sampling rate of $19.2 \mathrm{kHz}$ it would require $5.1 \times$ 106 floating point operations per second (FLOPS) in order to perform the required filtering. The AT32UC3C processor is capable of performing 15 MFLOPS meaning that the filtering alone would require $\sim 35 \%$ of the overall processing power. This was unacceptable and an alternative method had to be found. Therefore it was decided that every possible output value for the filter would be pre-calculated and stored in a lookup table in the processors flash memory, the filtering algorithm can then simply perform a lookup from this table to determine the filtered output values to send to the DAC. This brought the total processor usage down to less than $0.01 \%$. However, this processing power reduction was achieved at a cost of $32 \mathrm{kB}$ of non-volatile memory. 
Two options were evaluated for driving the DAC output, interrupts on their own, and DMA driven interrupts. In order to ensure the accurate timing requirements it was necessary to drive the DAC through the use of hardware interrupts, these were configured to trigger at the sampling rate of $19200 \mathrm{~Hz}$. Due to the use of FreeRTOS there are short periods of time that interrupts are disabled altogether. If the DAC is required to generate an output at time then it will be delayed, and in turn causes jitter in the sampling frequency; which is alleviated by reducing the interrupt frequency as much as possible. The on-board DMA module was used to achieve this and allowed for a single symbol worth of DAC outputs to be provided at once, thereby reducing the interrupt frequency, by the DAC oversampling rate, back down to the signal baud rate $-1.2 \mathrm{kHz}$ or $4.8 \mathrm{kHz}$. Ultimately the use of this method reduced the chances of sampling jitter occurring by the oversampling rate being applied.

The time domain results of the filtering process for the 1.2 $\mathrm{kHz}$ signal and $4.8 \mathrm{kHz}$ signals can be seen in Figure 8 respectively, and the frequency domain results in Figure 9. The oscilloscope time domain representations of the $1.2 \mathrm{kHz}$ signal can be seen to contain less high frequency components than the $4.8 \mathrm{kHz}$ signal as the waveform is more sinusoidal, with less sharp edges, this was expected and was due to the difference in oversampling rates for the two output baud rates.
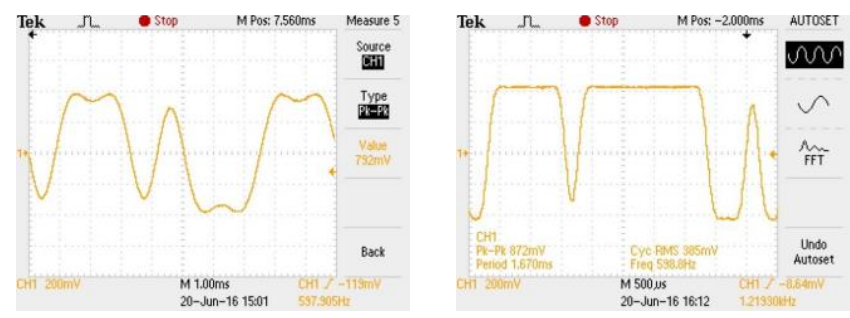

Figure 8: $1.2 \mathrm{kHz} \& 4.8 \mathrm{kHz}$ filter time responses
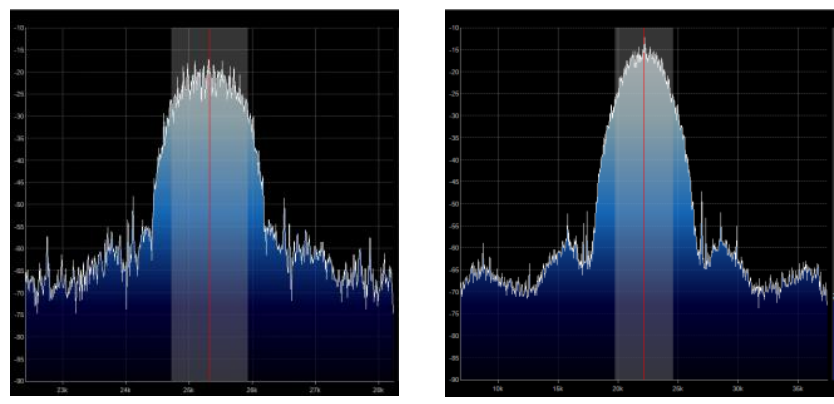

Figure 9: $1.2 \mathrm{kHz} \& 4.8 \mathrm{kHz}$ filter frequency responses

Overall, the use of digital filtering removed all unwanted transmission frequencies and it has been possible to achieve this with a total processor usage of $<0.1 \%$ and a memory usage of $32 \mathrm{kB}$. It would be possible for other projects to reduce the overall memory footprint through the use of fewer filter coefficients.

\section{AFSK UPLINK}

In addition to the requirement for the payload to be able to downlink telemetry, it was also a requirement that the payload could be controlled via telecommand, meaning an uplink channel was required. The AMSAT L-band uplink PCB demodulates a Frequency Modulated (FM) signal and presents the decoded baseband audio signal to ADCs on the processor. The baseband signal is then sampled by the ADCs on the processor such that the incoming data stream can be decoded in software. It was necessary to select a simple and reliable modulation strategy that could be decoded in real-time by an embedded processor with constrained memory and processor cycles available - for these reasons Audio Frequency Shift Key (AFSK) was selected for use. The Multimon C-code library already used in the project for decoding AFSK signals was already used in the project by earlier students [2]. This implementation however was not designed to operate on an embedded processor and as such required more modification before it could be used. In its original format, the Multimon library could be used to reliably decode received AFSK signals on the target processor, however, to achieve this it used $95 \%$ of the total processors cycles - as measured by FreeRTOS tools.

The library makes use of $1.2 \mathrm{kHz}$ and $2.2 \mathrm{kHz}$ waveforms to represent a 'mark' and a 'space' respectively. This means that the processor was required to sample at a fast enough rate in order to be able to determine the difference between these two waveforms. The sampling rate of the Multimon decoder was $22 \mathrm{kHz}$, or 10 times faster than the highest frequency contained within the baseband signal. A signal must be sampled at twice the highest frequency component in order to be fully reconstructed meaning that it was necessary to sample at a rate of $4.4 \mathrm{kHz}$. Testing was done at this rate with the findings that only $40 \%$ of packets were successfully decoded. This is believed to be due to sampling jitter and further possible imperfections in the Multimon decoding algorithm.

Thus the sampling frequency was systematically increased with a finding that a sampling rate of $8.8 \mathrm{kHz}$ allowed for a decoding rate of $70 \%$, in order to reach a decoding rate of $99 \%$ the rate was increased to $11 \mathrm{kHz}$. This represented a halve in the sampling frequency originally used in the library and brought the overall processor usage down from $95 \%$ to $45 \%$, a large reduction in processor cycles but still insufficient to abide by the ESA processor usage standards of $50 \%$ when other threads are considered; and thus further processor cycle reduction was necessary.

The Multimon library operates through the use of floating point mathematics whereas the inputs provided from the ADC are in fixed point form. On embedded processors, floating point operations typically take much longer than fixed point operations. Thus, in order to improve the performance of the Multimon library, it was converted to operate using fixed point, the final result of which being that the overall processor usage for decoding was reduced to 
$28 \%$. This is within the usage boundaries laid out by ECSS and was therefore deemed as acceptable.

In order to prove the behaviour of our corrected Multimon implementation, a software test harness was created using $\mathrm{C}++$ in the xUnit Google Test Framework [8], a bespoke AFSK encoder was then developed within the harness, this encoder took a binary input stream and created an AFSK signal at the correct rate for the Multimon decoder sampling frequency. The use of this test harness meant that the library functionality could be tested without having the hardware present and without an RF commanding chain to send AFSK packets to the payload, this allowed for the library to be tested in isolation from the rest of the system. The input and output of this encoder can be seen in Figure 10.

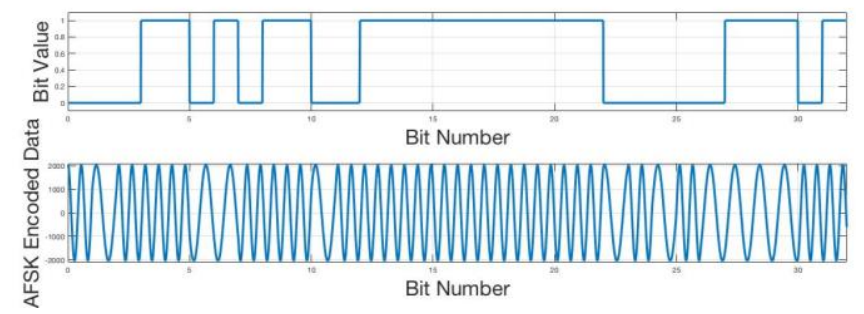

Figure 10: Test Harness for AFSK using xUnit Google Test Framework

\section{Thread Reliability \& Performance}

Understanding and testing of any system is key to ensuring long term successful operation and finding bugs. Therefore a large focus was placed upon testing through-out this project, in addition heavy profiling of the processor performance and loading was performed. In order to better analyse the performance of the system Tracealyser from Percepio was used, this allowed for visualization of the system operation to an otherwise unobtainable degree [9].

A key requirement of the ESA software development standards is that average processor usage is kept to below $50 \%$ to allow for peaks in required processing power. It was expected that the uplink thread would consistently use the highest processor percentage; the operations performed in this thread however are a repetitive mathematical process and will therefore always require the same amount of processing time. It was then expected that the downlink thread would create large spikes in processor usage as the periodic FEC process is mathematically intensive. It was essential to ensure that the processor could cope with both the background processing requirements and also with the peaks demands in processing power. Figure 11 is taken from Tracalyser where each thread is represented by a different colour. It can be seen that approximately $25 \%$ of processor cycles are spent running the uplink thread and that this percentage only varies slightly with time.

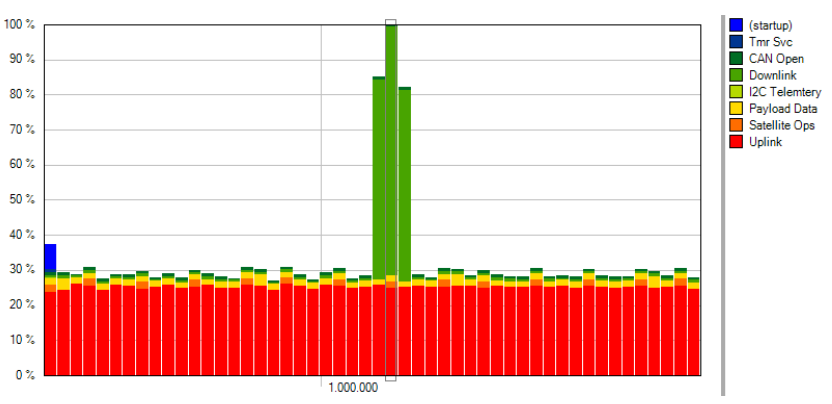

Figure 11: Percepio Tracealyzer output visualizing processor loading over time per thread

It can also be seen that the downlink thread requires the most processing power, however, it only requires this power when it is performing FEC on a packet to be transmitted once every 1.25 seconds when operating at the maximum downlink baud rate. The processing power required to perform FEC reaches $100 \%$ which would typically be cause for concern in a system, however, through the use of Tracalyser it was possible to ensure that FreeRTOS was allowing for pre-emptive multitasking to take place and thereby ensuring that all threads and interrupts were still executing at the required time.

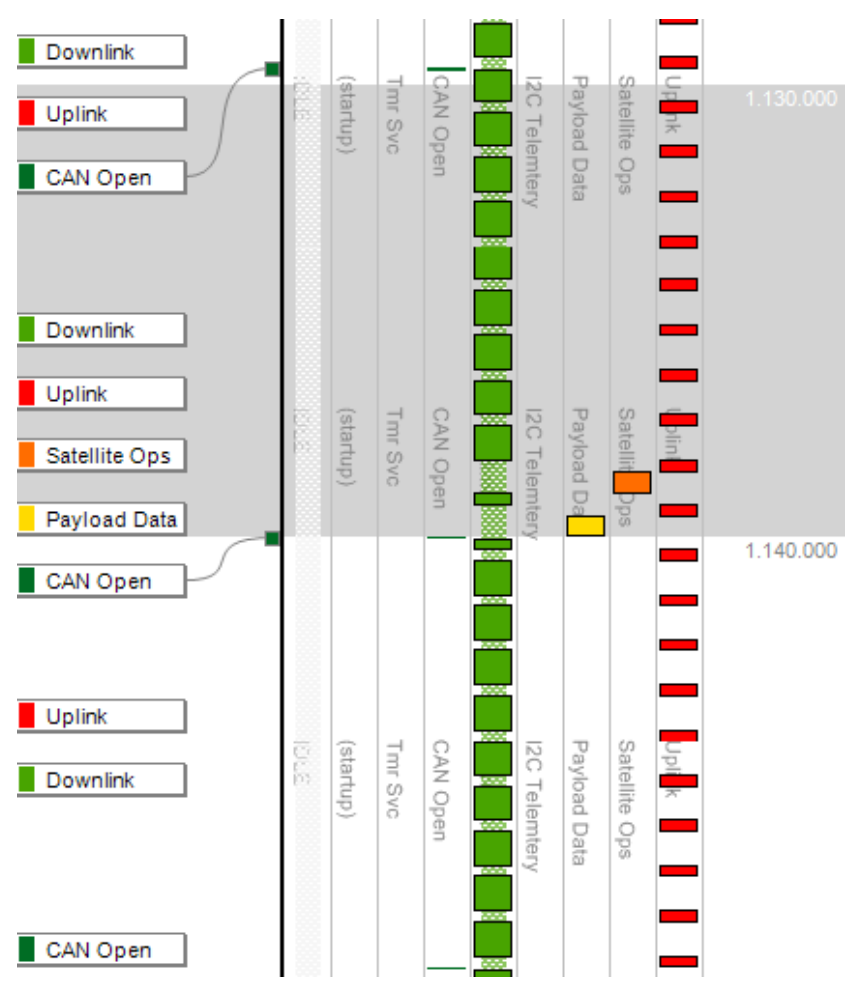

Figure 12: Percepio Tracealyzer Timing execution diagram showing per thread timing

Figure 12 is a timing execution diagram that shows when threads are executing and for how long. It also covers the same time period when the uplink thread is performing FEC and causing $100 \%$ usage of the processor in Figure 11. It can be seen that although the downlink thread is consuming the majority of the processor cycles, the other system threads are still being allowed to run correctly. This means 
that through the use of FreeRTOS the overall system performance can still be guaranteed even with the processing power peak requirements imposed by the use of mathematically intensive FEC techniques. The full timing diagram can be used to determine that the time required under normal processor loading conditions to perform FEC of a single packet is $80 \mathrm{~ms}$, whereas the requirement when operating at the maximum baud rate is 1.25 seconds, therefore it would be possible for this processor to operate at a higher downlink baud rate - from the processor usage and timing data shown it is expected that $9.6 \mathrm{kbps}$ could comfortably be achieved on this processor while further consideration would have to be given to a data rate of 19.2 kbps.

There are two system interrupts used for sampling, one for sampling the incoming AFSK uplink signal and one for providing samples to the BPSK downlink modulator. The performance of the uplink and downlink channels is heavily dependent the timing of these interrupts, sampling at the incorrect point in time can cause distortions to the signals received and transmitted, degrading the overall performance of the system. So it was essential to, again, prove the timing of these interrupts and also to quantify any sampling period jitter.

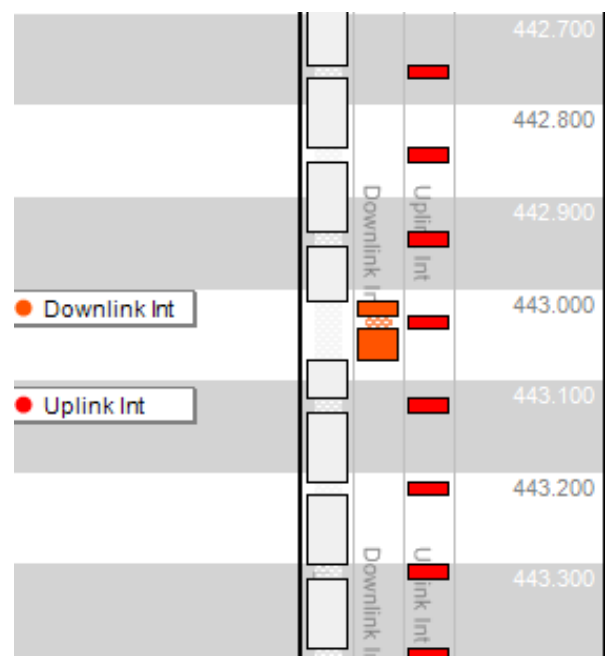

Figure 13: Downlink \& Uplink Interrupt Service Routine function times showing colliding

Figure 13 shows both sampling interrupts, the downlink sampling ISR can be seen to be sampling at approximately $10 \%$ of the speed of the uplink sampling ISR, $1.2 \mathrm{kHz}$ and $11 \mathrm{kHz}$ respectively. The execution period of both interrupts can also be seen to be different, 30 us for the downlink ISR and 15 us for the uplink. When the analysis of the processor performance was carried out, the system was operating as in Figure 12, whereby after $442.8 \mathrm{~ms}$ two interrupts occur at the same time. This has caused the uplink sampling ISR to have to wait for the downlink ISR to finish, this in turn caused a delay in execution of 30 us which equated to $33 \%$ of the uplink sampling period. This ISR collision and jitter will have the effect of preventing the signal being decoded at the Nyquist frequency and cause the sampling rate relative to the highest frequency baseband component to require increasing. Once this problem had been observed, it was possible to make changes in FreeRTOS to counteract this problem; as in Figure 14. This was achieved through enabling nested interrupts, thereby allowing the uplink ISR to interrupt the downlink ISR, and meaning that the downlink ISR would instead become jittered if both interrupts occurred at the same time.

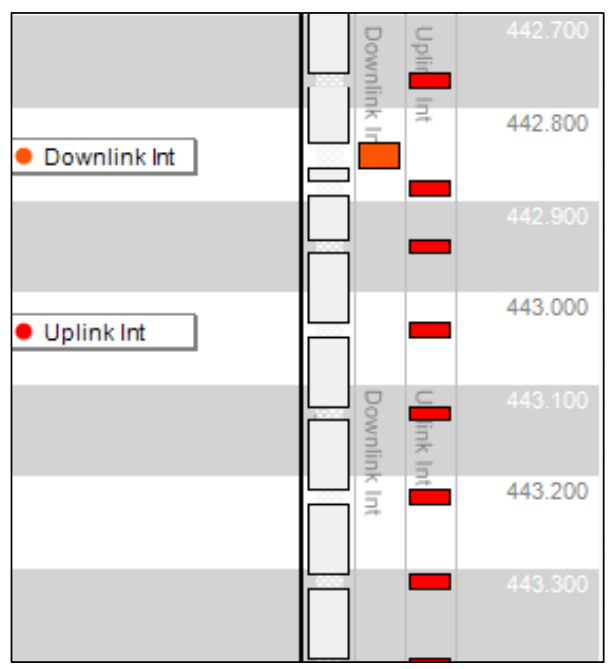

Figure 14: Downlink \& Uplink Interrupt Service Routine function times showing no collision

Due to the slower sampling rate of the downlink and the shorted period of the uplink ISR this resulted in a sampling jitter of only $1.8 \%$ of the sampling frequency in $1.2 \mathrm{kbps}$ mode and $7.2 \%$ in $4.8 \mathrm{kbps}$ mode. If future work were to be performed increasing the downlink baud rate then a key area of consideration would be the frequency of interrupt collision and the associated jitter. A final analysis of the RAM used showed that of the $64 \mathrm{kB}$ originally available 17 $\mathrm{kB}$ remained.

\section{Project Experiences \& Challenges}

Over the years, there have been many team members; both in AMSAT-UK and with university students. Three sets of students have led to the delivery of a satellite payload - over 3 'university cycle' years. Real world issues in skilling up students only to watch them leave to new studies or jobs means that long project durations and deadlines are incompatible to university timetables; something the team have struggled with.

There is also further compromise on documentation requirements and the industry standard output of a professional engineer working $40 \mathrm{hrs}$ a week cannot be compared to student availability. As such, there is scope to deliver a broad set of engineering best practice parameters, which allow for design flexibility within a range of defined acceptable practices / procedures.

Communication between different working groups has been critical for continued motivation this mission and the 
payload, as with other distributed projects. Project updates at top level can provide continuing motivation for lower level activities and payload teams.

Attention to detailed project management is also needed and a weekly team phone call and constant student meetings have pushed forward this project. As each small and new group of students will have limited appreciation and experience to the overall scope of a project which has been largely down to the AMSAT team to advance the project locally. The team encourages focus on the detail and that advice is sought when working on unfamiliar topics. This increases communication skills and adds reality where unjustified self-belief can predominate if left alone.

Despite these issues, each student has found real value in working with the experienced AMSAT team on a real mission. We note the following feedback: "it has been great to get the opportunity to work on something that is going to go into orbit, that fact has been really motivational throughout the year. It has also been good to be able to collaborate with AMSAT and Sitael as it gives an exposure that would otherwise have been missed." Each U.K. student involved in the project has gone on to $\mathrm{PhD}$ studies.

\section{SUMMARY}

The overruling theme throughout this project development has been on maximizing quality, this has been primarily through the use of the TDD process which has shown itself to be invaluable on several occasions, both through allowing development away from radio hardware and also for ensuring interfaces have been properly met - it is a thorough recommendation that this process is used for any similar developments in the future.

The AT32UC3C processor has been critically evaluated to show its performance when performing processor cycle intensive communications techniques, such as forward error correction, with a finding that the processor can easily cope with the $4.8 \mathrm{kbps}$ data rate required of it. It is also a recommendation that the processor could be evaluated further for use at a higher data rate of $9.6 \mathrm{kbps}$ or possibly even $19.2 \mathrm{kbps}$.

Typically digital filtering is done through the use of Fourier Transforms or convolution at run-time, it was the finding of this work that the AT32UC3C was not capable of performing filtering in this way and that embedded software development techniques would have to be applied in order to enable the filtering to be done - this was in the form of a lookup table. This table has proven itself to have a large memory footprint however and the number of filters required to be stored should be heavily considered before selecting a processor.

An invaluable tool has been found in Tracelyser from Percepio, allowing for the processor behavior to be placed under great scrutiny and for typically unobserved errors to be seen. However, more issues could have detected through its use were it deployed from the projects start, therefore it is suggested that a tool such as this be used throughout a projects development.

The use of radiation hardened components was not an option for the budget of this mission so other techniques had to be examined. Whilst standard industry techniques were implemented such as the user of triple modular redundancy and memory scrubbing, a novel technique was also developed by combining triple modular redundancy with the operating systems kernel behavior. The end result being a technique offering stack variables a high percentage of protection, but also collecting the statistical data associated with the technique such that its efficacy can be established for use in future missions.

\section{ACKNOWLEDGEMENTS}

The authors would like to acknowledge the AMSAT team: Duncan Hills, Dave Johnson, Howard Long and Wouter Weggelaar. There are also many students which have made real contributions to the project: Eduardo Theves Lourenco, Viktor Lopes de Castro Martinelli, Ben Clewer, Ben Chapman, Andre Vitor Celkevicius. Thanks also go to Percepio for their time and tools. Finally, thanks to ESA, ALMAspace and Sitael staff for their continued efforts in the ESEO mission.

\section{REFERENCES}

[1] ESA, "Call of Interst for ESEO and ESMO Project," $2802 \quad 2008$ [Online]. Available: http://www.esa.int/Education/Call_for_interest_for_ES EO_and_ESMO_projects. [Accessed 2110 2016].

[2] E. Önal, "Multimon Github," 0804 2016. [Online]. Available: https://github.com/EliasOenal/multimon-ng. [Accessed 2110 2016].

[3] European Cooperation for Space Standardization, "ECSS-E-ST_40C," ECSS, 2009.

[4] P.Karn, "Proposed Coded AO-40 Telemetry Format," $1212002 . \quad$ [Online]. Available: http://www.ka9q.net/papers/ao40tlm.html.

[5] S.B.Damelin, "Compressive Sampling," in The Mathematics of Signal Processing, Cambridge, Cambridge Press.

[6] A. Devices, "MT-107 Oversampling Interpolating DACs," Analogue Devices, 2016.

[7] A. Fisher, "Raised Cosine Filters," York University.

[8] G. Inc., "Google Test Framework," 22082016. [Online]. Available: https:/github.com/google/googletest/.

[9] P. AB, "Tracealyzer," [Online]. Available: http://percepio.com/tz/. 


\section{BIOGRAPHY}

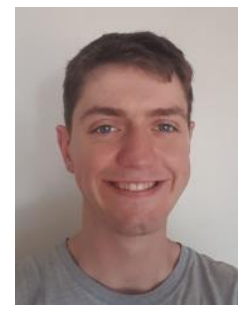

Pete Bartram received an $M S c$ in Space Engineering from the University of Surrey in 2016 and a BEng in Digital Electronics, Computing and Communications from Brighton University in 2012. He has also spent 5 years within industry developing embedded software for safety critical systems. Currently he is pursuing a PhD at the University of Southampton with a focus on large scale simulations of Astrodynamics and Planetary Formation.

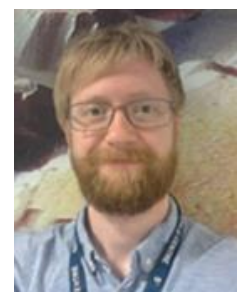

Dr Christopher P. Bridges (BEng, 2005; PhD 2009) leads the On-Board Data Handling (OBDH) research group within Surrey Space Centre (SSC). He researches software defined radios, real-time embedded systems, agent computing, Java processing, multi-core processing in FPGAs, and astrodynamics computing methods in many spaceflight payloads. In 2013, he designed, built and still operates the UK's first CubeSat (STRaND-1) with SSTL and now contributes towards computing hardware and software with SSTL, on ESA's ESEO mission and also the NASA-JPL/CalTech AAReST mission.

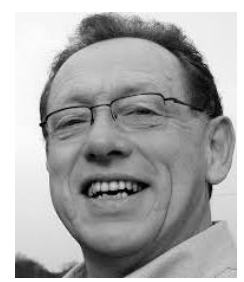

Graham Shirville has been a radio amateur for many years and has studied space technology since the beginning of the space age. $\mathrm{He}$ established his first groundstation, to communicate via the Phase 3 OSCAR spacecraft which operated in GTO, during the 1980s and 1990s. Later he was involved with the construction and launch of the SSETI Express spacecraft in 2005. Currently he is working with the FUNcube team to provide additional spacecraft to increase STEM outreach from space.

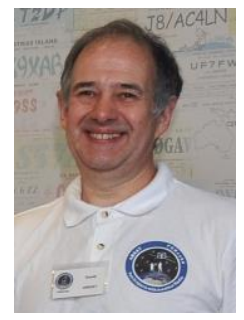

David Bowman is an RF engineer working with AMSAT as a volunteer. Having completed his studies at Brooklands and Paddington Technical Colleges, he taught Electronics at FE before starting a small business specialising in designing and supplying point to point communications equipment. An AMSAT member since the late 1970s, he worked on SETTI Express from ESA Education (2005). Since then he has contributed to the FUNcube-1 educational outreach mission (launched 2013) as well as UKube-1 and QB50p-1. He is currently working on $L$ band and VHF communication systems for ESEO. He teaches Physics part time in a West London secondary school and rescues ex-racing Greyhounds. 